\title{
Tandem DNAzymes for mRNA cleavage: choice of enzyme, metal ions and the antisense effect
}

\author{
Feng Wang, ${ }^{a}$ Runjhun Saran ${ }^{a}$, and Juewen Liu ${ }^{*}$
}

${ }^{a}$ F.W. and R.S. contributed equally to the work. Department of Chemistry, Waterloo Institute for Nanotechnology, University of Waterloo, Waterloo, Ontario, N2L 3G1, Canada. Fax: 519 7460435; Tel: 5198884567 Ext.38919; E-mail: liujw@ uwaterloo.ca.

The final publication is available at Elsevier via http://dx.doi.org/10.1016/j.bmcl.2015.02.032 (C) 2015. This manuscript version is made available under the CC-BY-NC-ND 4.0 license http:// creativecommons.org/licenses/by-nc-nd/4.0/ 


\begin{abstract}
The concept of DNAzyme-based gene silencing via mRNA cleavage was proposed over twenty years ago. A number of studies regarding intracellular gene silencing have been reported as well. However, questions have been raised regarding the lack of enzyme activity in physiological buffer conditions and it is being doubted that in the previously reported studies, gene silencing might be simply due to an antisense effect. In this work, two classical DNAzymes for RNA cleavage are studied using both chimeric substrates and extracted mRNA. We concluded that the activity of the 8-17 DNAzyme is much higher than that of 10-23 in the same conditions. To illustrate and compare the effect of specific cleavage versus antisense effect in the best possible way, we used tandem DNAzymes. Specific mRNA cleavage occurred with $\mathrm{Zn}^{2+}$, while with $\mathrm{Mg}^{2+}$, even the inactive control DNAzymes showed a similar response, suggesting that the antisense effect might be the dominating phenomenon causing gene silencing. This study has thus clarified the choice of DNAzyme sequence, the effect of metal ions and a potential source of antisense effect.
\end{abstract}


A number of strategies have been developed to selectively cleave mRNA for anti-viral and anticancer applications. ${ }^{1}$ For example, antisense DNA relies on DNA oligomers to form a duplex with target mRNA and recruit RNase $\mathrm{H}$ to cleave the RNA strand. ${ }^{2}$ With siRNA, the cellular RNA interference pathway is harnessed and the target mRNA is efficiently degraded. ${ }^{3}$ Ribozymes are RNA-based enzymes and many naturally occurring ribozymes have RNA cleavage activity. A few artificial ribozymes have also been engineered to cleave mRNA without the involvement of protein components. ${ }^{4}$ However, RNA has low stability and is easily degraded. ${ }^{5}$ DNAzymes are DNAbased enzymes. ${ }^{6-10}$ Since first report in $1994,{ }^{11}$ DNAzymes have been proposed to cleave mRNA. ${ }^{12-14}$ DNAzymes can execute the same catalytic function as ribozymes but are more stable, cost-effective and easier to label. ${ }^{6-10}$

In 1997, Santoro and Joyce reported two general-purpose RNA-cleaving DNAzymes that cleave all-RNA substrates with efficiency comparable to that of protein enzymes. ${ }^{7,}{ }^{15}$ Out of the two, the 10-23 DNAzyme has been widely studied for intracellular mRNA cleavage. ${ }^{13,}$ 16-18 This is probably because of its excellent substrate generality, it cleaves any purine/pyrimidine junctions, ${ }^{7}$ which offers tremendous freedom to target many regions of interest within an mRNA. The other one, the 8-17 DNAzyme, has been more researched upon for metal detection since it has excellent activity in the presence of low concentrations of $\mathrm{Pb}^{2+} .{ }^{19}$ At the same time, it is also studied as a model DNAzyme due to its recurrence in many different in vitro selections and high catalytic efficiency. ${ }^{20-25}$ Examples of using the 8-17 DNAzyme for gene silencing are also known. ${ }^{18,26}$

While many examples of DNAzyme-based intracellular mRNA cleavage are reported, ${ }^{13}$ concerns have also been raised that the 10-23 DNAzyme is basically inactive with physiological concentrations of free $\mathrm{Mg}^{2+} \cdot{ }^{27}, 28$ Biochemical and spectroscopic studies suggest that previously reported 10-23 DNAzyme induced gene silencing might be simply due to an anti-sense effect 
instead of DNAzyme-induced cleavage. ${ }^{29,}{ }^{30}$ Since most of the previous gene silencing work only measured the final mRNA or protein concentration, while the step-by-step reaction processes were not followed, the exact role of the DNAzymes remains elusive. ${ }^{13}$ To move forward, a clear understanding on the reaction steps of DNAzyme catalysis is needed. Herein, we perform a few comparisons between the 10-23 and 8-17 DNAzymes and aim to answer the following questions related to mRNA cleavage: 1) the optimal DNAzyme to be used, 2) the DNAzyme activity in the presence of different metal ions, and 3) the antisense effect versus DNAzyme-induced cleavage.

The secondary structures of the 8-17 and 10-23 DNAzymes are shown in Figure 1A and 1B respectively. For each DNAzyme, the substrate strand contains a single RNA linkage that can be cleaved by the enzyme strand in the presence of divalent metal ions. In the original paper, the 10-23 DNAzyme was described to cleave $r R \cdot Y(R=A, G ; Y=T, C)$ junctions (Figure 1B has an rG.T junction). ${ }^{7}$ The 8-17 DNAzyme cleaves the rA.G junction, and appeared to be less general during its initial studies. However, after extensive selection and mutation work, the Li group discovered that the 8-17 DNAzyme can cleave essentially all the 16 possible dinucleotide junctions, although the rate varies over 5 orders of magnitude for these junctions. ${ }^{21}$ By introducing mutations in the enzyme, the 8-17 DNAzyme can be optimized for each type of junction. It was recently proposed that the 8-17 and 10-23 DNAzymes might be the same enzyme. ${ }^{31}$ In other words, the 1023 DNAzyme might be a mutant of the $8-17 .{ }^{31}$ This conclusion was made based on mutation studies. We are interested in testing their activity in the presence of different metal ions. 

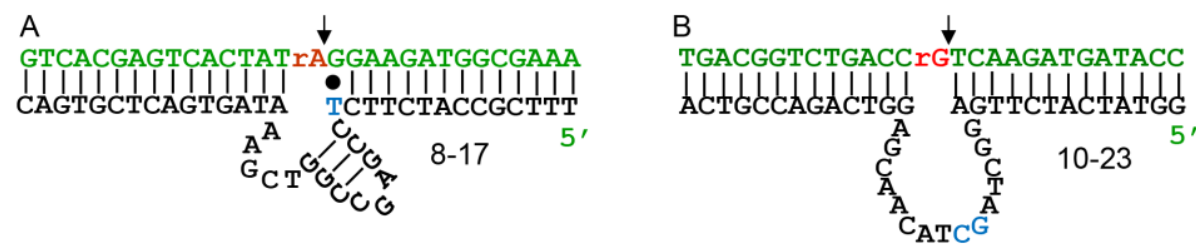

C

10-23 DNAzyme + $0.1 \mathrm{mM} \mathrm{M}^{2+}$

- Mg Ca Ba Sr Zn Mn Ni Co Cd Pb
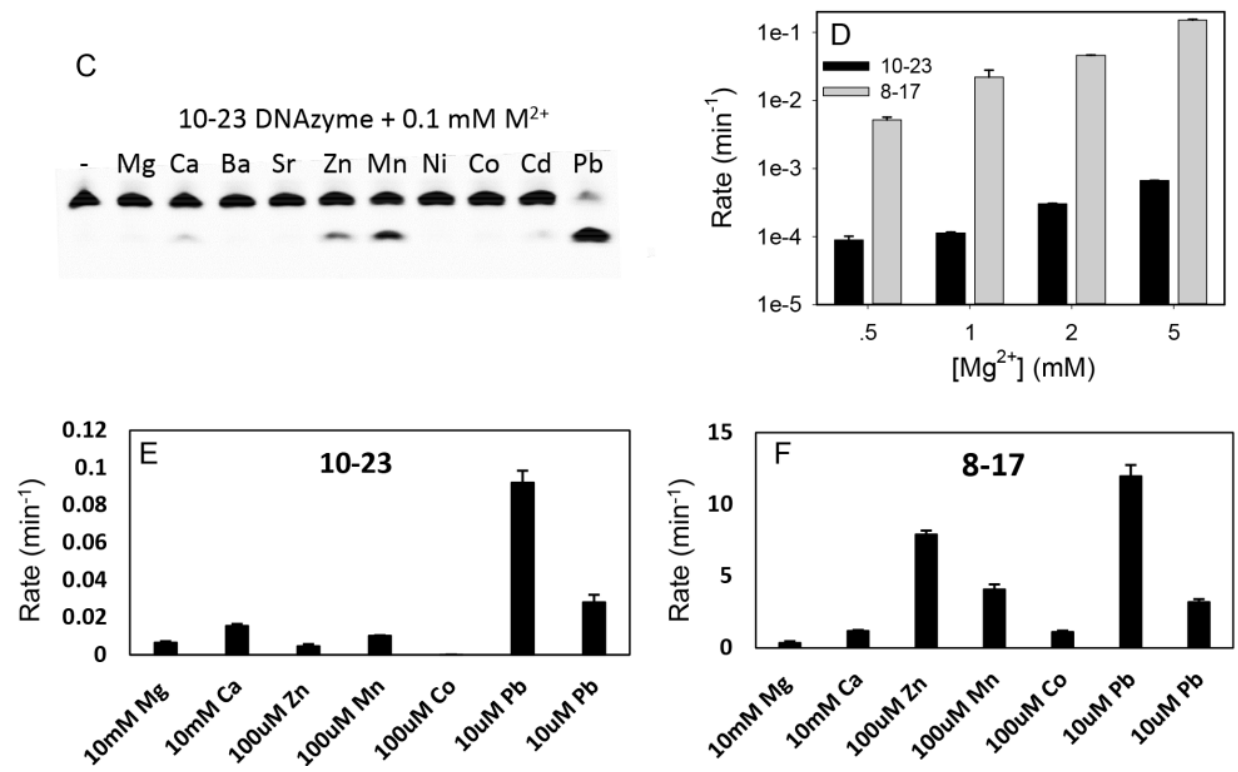

Figure 1. The secondary structure of the (A) 8-17 and (B) 10-23 DNAzymes. By mutating the T (in blue) to $\mathrm{C}$ for the $8-17$ or $\mathrm{C}$ to $\mathrm{T}$ and $\mathrm{G}$ to $\mathrm{C}$ for the $10-23$, inactive DNAzymes are generated. (C) A gel picture of the 10-23 DNAzyme cleavage in the presence of $0.1 \mathrm{mM}$ divalent metal ions. Cleavage rates of (D) the two DNAzymes in the presence of different concentrations of $\mathrm{Mg}^{2+},(\mathrm{E})$ the 10-23 DNAzyme and (F) the 8-17 DNAzyme in presence of different divalent metal ions at various concentrations. All the reactions were carried out at $\mathrm{pH} 7.4$ except for $\mathrm{Pb}^{2+}$ at $\mathrm{pH} 6.0$ (the last bar in Figure 1E and 1F).

Divalent metal ions are an indispensable part of DNAzyme catalysis, ${ }^{32-34}$ and a key concern raised is the activity of the $10-23$ DNAzyme in physiological $\mathrm{Mg}^{2+}$ concentrations. ${ }^{27,}{ }^{28}$ To fully understand this, we first reacted the 10-23 DNAzyme with various other divalent metals for $1 \mathrm{~h}$ and the products were analyzed using gel electrophoresis (Figure 1C). Since one end of the 
substrate was labeled with a carboxyfluorescein (FAM), a darker lower band indicates more cleavage. $\mathrm{Pb}^{2+}$ induced the most cleavage, which was the same as the 8-17 DNAzyme. ${ }^{23}$ To have a quantitative comparison, we further measured the enzyme rate in the presence of these metal ions (Figure $1 \mathrm{E}$ ). $\mathrm{Pb}^{2+}$ has the highest activity followed by transition metals such as $\mathrm{Mn}^{2+}$ and $\mathrm{Zn}^{2+}$ (note that $\mathrm{Mg}^{2+}$ and $\mathrm{Ca}^{2+}$ were used at a much higher concentration). It is not surprising that the 10-23 DNAzyme has the highest activity with $\mathrm{Pb}^{2+}$ since both DNAzymes share the same conserved nucleotides. ${ }^{31}$ If we compare both, the 8-17 DNAzyme exhibits a similar trend but is $\sim 100$-fold more active at the same metal concentrations (Figure 1F, note the difference in the scale of the $y$-axis). The significance of the similar trend observed in the above experiment also lies in the fact that it supports the theory that these two DNAzymes might belong to the same family and undergo the similar cleavage mechanism.

For mRNA cleavage, the most important metal ion is $\mathrm{Mg}^{2+}$, since this is the most abundant divalent metal ion inside cells. ${ }^{35}$ Other metal ions, although yield faster cleavage, are not present as free ions in cells at useful concentrations. ${ }^{35}$ Next we compared the DNAzyme activity in the presence of low concentrations of $\mathrm{Mg}^{2+}$ (Figure 1D). Both DNAzymes showed $\mathrm{Mg}^{2+}$ concentration-dependent activity. Again, the 8-17 was 100-fold more active. For mRNA cleavage, we need to use the most efficient DNAzyme. Therefore, the 8-17 DNAzyme is likely to be a better choice. Among the four junctions that the 10-23 DNAzyme can cleave, this rG.T junction is a relatively easily cleavable one. For example, when an rA.C junction was used, barely any cleavage was observed even with $500 \mathrm{mM} \mathrm{Mg}^{2+}$ (Figure S1, ESI). Therefore, it is also true for the 10-23 DNAzyme that its cleavage activity for every junction is not the same.

The above work was carried out using RNA/DNA chimeric substrates, and the activity for cleaving pure RNA might be different. To further compare the DNAzymes for RNA cleavage, we 
used mRNA as substrate. Since cleaving mRNA inside cells requires DNAzyme delivery and it is more difficult to draw quantitative conclusions, we started with extracted mRNA. We chose to target the mRNA for glyceraldehyde 3-phosphate dehydrogenase (GAPDH), which is an abundant house-keeping gene. After PCR primer optimization, we fixed a region of the mRNA to target (see Figure S2 for real-time PCR quantification). In this mRNA fragment (Figure 2A), two cleavage sites were identified, one to be targeted by the 10-23 DNAzyme (called 23E1, see Figure S3 in ESI for sequence) and the other by the 8-17 DNAzyme (17E1). These two sites are only four nucleotides apart, therefore the effect from mRNA secondary structure should be minimal.

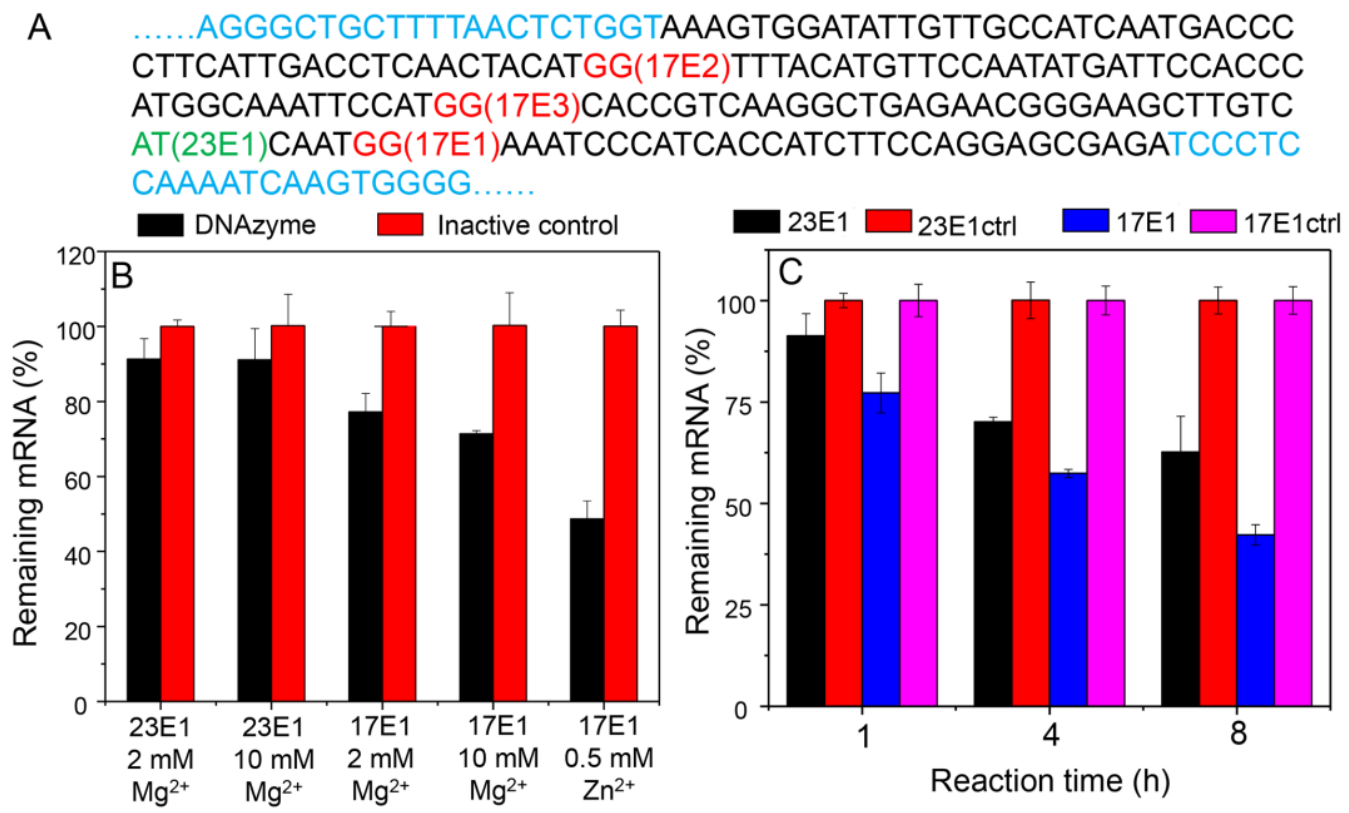

Figure 2. (A) A fraction of the GAPDH mRNA sequence with the PCR primer binding region in blue. The DNAzyme targeted cleavage junctions are shown before the parenthesis indicating the 
name of the enzyme. (B) Cleavage of the mRNA by 17E1 or 23E1 in the presence of different metal ions for $1 \mathrm{~h}$. (C) Cleavage by the different DNAzymes as a function of time with $2 \mathrm{mM} \mathrm{Mg}^{2+}$.

The extracted total RNA was mixed with each DNAzyme in presence of $2 \mathrm{mM} \mathrm{Mg}^{2+}$ for 1 h. After reaction, the products were reverse transcribed into cDNA and then quantified using realtime (RT) PCR, which is the typical protocol for mRNA quantification in gene silencing work. To ensure that the measurement was made on cleavage instead of artifacts, mutated control DNAzymes were also included (mutated nucleotides are indicated in blue in Figure 1A, B). These mutated nucleotides are known to be critical for activity. To highlight the effect of cleavage, all the samples were normalized against their control DNAzyme (Figure 2B). The decrease of mRNA was only $\sim 10 \%$ for the 23E1 DNAzyme and this difference was statistically insignificant considering the error bars. On the other hand, 20-30\% decrease was achieved with 17E1 using the same $\mathrm{Mg}^{2+}$ concentration and $\sim 50 \%$ decrease was achieved with $0.5 \mathrm{mM} \mathrm{Zn}{ }^{2+}$. Next, we studied time-dependent mRNA cleavage using $2 \mathrm{mM} \mathrm{Mg}^{2+}$ (Figure 2C). For all the samples, we observed more cleavage with time and the 17E1 always exhibited more cleavage. This comparison also supports that the 8-17 DNAzyme can be better at cleaving mRNA.

After determining the best enzyme, we next aim to further increase the cleavage yield. Since an mRNA is quite long and cleavage at any point destroys its function, a method to increase cleavage is to use a few DNAzymes simultaneously to target different positions along the mRNA. To test this, we designed three 8-17 DNAzymes (named 17E1, 17E2 and 17E3, Figure 2A). For each DNAzyme, a corresponding inactive mutant was used as control. These DNAzymes target the GG dinucleotide junctions to achieve a high cleavage activity. ${ }^{21}$ Since the PCR primers cover the whole region, any cleavage should result in the signal suppression. To avoid artifacts due to 
DNAzyme concentration, all the samples contained the same amount of total DNAzyme (e.g. 100 $\mathrm{nM}$ of each of 17E1, 2, and 3 when all were used together; or $150 \mathrm{nM}$ of both 17E1 and 2 when only the two were used together; or $300 \mathrm{nM} 17 \mathrm{E} 1$ if it was the only DNAzyme used). In addition, a sample without DNAzyme treatment was also analyzed as the reference standard for comparison. Here, we aim to compare both non-specific (e.g. antisense) and specific (cleavage) effects of the added DNAzymes, and the data was normalized against the untreated samples (instead of control

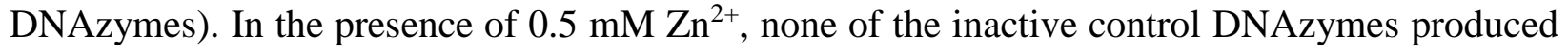
much cleavage and nearly 100\% mRNA remained for all the samples (Figure 3A, red bars), suggesting that the DNAzymes did not cause non-specific effects. In most cases, samples with the active DNAzymes (black bars) produced more mRNA cleavage compared to the control DNAzymes. We also observed that more the number of DNAzyme added, more was the inhibition effect (Figure 3A, the average of the two combined has $\sim 34 \%$ more inhibition than the average of the three individual ones; the three combined has the largest inhibition effect). Therefore, the tandem DNAzymes can promote mRNA cleavage.

When the same reactions were carried out in the presence of $2 \mathrm{mM} \mathrm{Mg}^{2+}$, however, a completely different pattern was observed (Figure 3B). The full mRNA concentration measured from RT-PCR dropped significantly as the number of DNAzymes increased (red bars). Since these are inactive DNAzymes, the observed decrease in the RT-PCR signal cannot be attributed to cleavage, and we attribute this to a simple antisense effect. On the other hand, the signal from the active DNAzymes (black bars) are similar to that from the inactive controls (red bars) in most cases, especially when mixed DNAzymes were used, suggesting that the specific cleavage related events were insignificant. In both studies, 17E3 (the last set of bars) showed a similar activity between the real enzyme and control, indicating that targeting this position by the DNAzyme was 
ineffective. This might be related to some stable secondary structures in the mRNA, hindering DNAzyme binding.

When multiple DNAzymes are used in tandem, two effects may have taken place. First, after hybridizing with the first DNAzyme, hybridization with the subsequent ones could become easier, since the first DNAzyme may break possible local secondary structures. Thus, if cleavage reaction can take place, there should be synergistic effects to increase the level of cleavage. Second, once such DNAzyme binding structures are formed, especially in tandem, they may pose a steric barrier for subsequent reactions to measure mRNA, namely the reverse transcription reaction. This blocking effect can be described to be a type of antisense effect.

In this study, $\mathrm{Zn}^{2+}$ was used because it is more active at low metal concentrations. So, its effect on ionic strength change is small and we can better probe the DNAzyme cleavage activity. $\mathrm{Mg}^{2+}$ was used because it is believed to be the most relevant metal inside cells. $\mathrm{Mg}^{2+}$ is also highly effective in stabilizing DNA duplex, which is consistent with the observed antisense effect. The antisense effect of DNAzymes is typically referred to the recruitment of RNase $\mathrm{H}$ inside cells, but this study suggests that other types of antisense effects along the whole assay process are also possible.
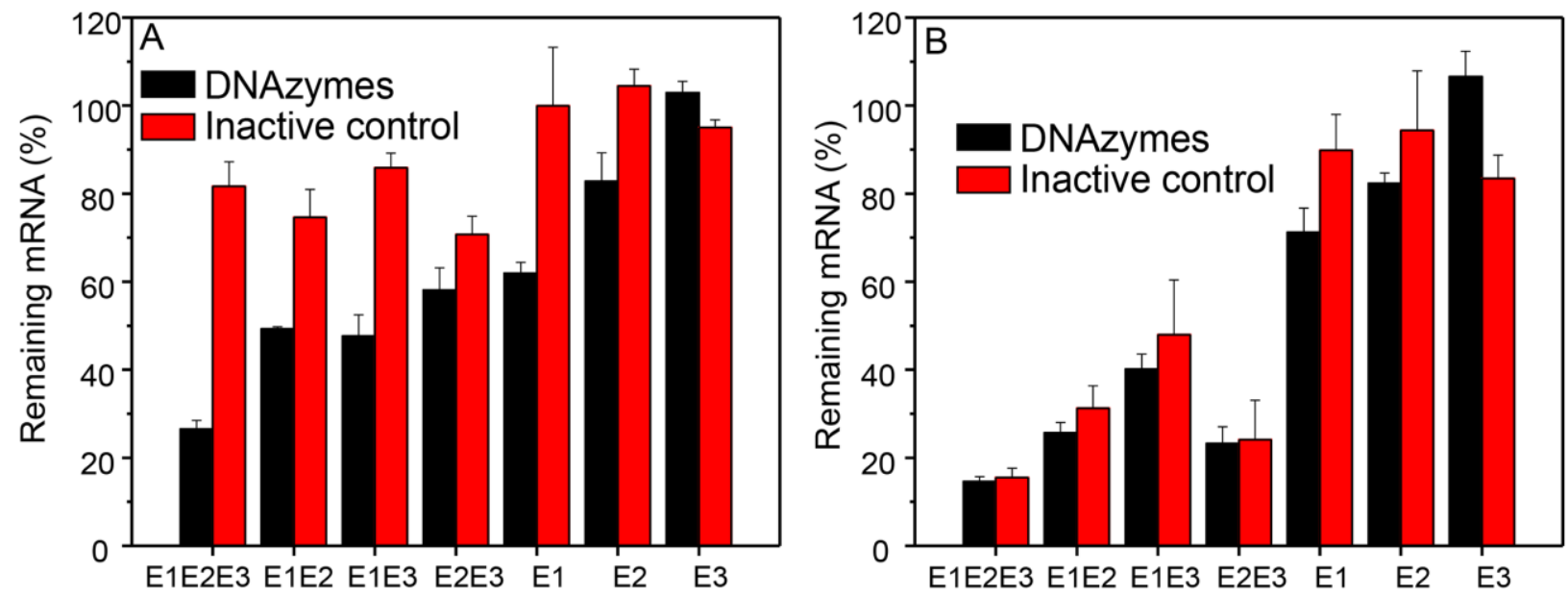
Figure 3. Cleavage of extracted mRNA using tandem 8-17 DNAzymes in the presence of (A) 0.5 $\mathrm{mM} \mathrm{Zn}^{2+}$ and (B) $2 \mathrm{mM} \mathrm{Mg}^{2+}$ for $1.5 \mathrm{~h}$. Total DNAzyme concentration $=300 \mathrm{nM}$. Each DNAzyme is compared with an inactive control sequence. The difference between red and black bars accounts for mRNA cleavage. The suppression of the red bars is due to an antisense effect.

In summary, we compared two classical DNAzymes with respect to their RNA cleavage activity. It is crucial to have control DNAzymes in mRNA cleavage studies. Otherwise, one cannot draw a conclusion that gene silencing is due to DNAzyme-induced cleavage instead of antisense effect. Second, while both DNAzymes have measurable activity in the presence of physiological concentration of $\mathrm{Mg}^{2+}$, the 8-17 DNAzyme is $~ 100$-fold faster than the particular 10-23 DNAzyme for cleaving chimeric substrate. Since they are active also with other divalent metal ions (e.g. $\mathrm{Zn}^{2+}$ ), their actual activity in cells might be higher. Third, in the original paper, ${ }^{7}$ a major difference between these two DNAzymes is the substrate cleavage junction. The efficiency for cleaving different junctions by the 8-17 DNAzyme has been systematically studied. ${ }^{21}$ We show here that the 10-23 DNAzyme also cleaves different junctions quite differently. If we accept the notion that both DNAzymes belong to the same family, and each junction may have its own optimal substrate junction sequence for cleavage, these two classic DNAzymes are just two examples of this DNAzyme family. Instead of focusing on which between the two should be used, it is probably more important to look at the dinucleotide junction at the targeted cleavage site, and then to find the optimal enzyme sequence. ${ }^{21}$ 


\section{Acknowledgements}

Funding for this work was from the University of Waterloo, Ontario Ministry of Research \& Innovation, and the Natural Sciences and Engineering Research Council (NSERC) of Canada.

\section{Supplementary data}

Supplementary data (materials and methods, additional biochemical assays) associated with this article can be found, in the online version, at http://dx.doi.org/10.1016/j.bmcl.

\section{References}

1. Deleavey, Glen F.; Damha, M. J. Chem. Biol. 2012, 19, 937.

2. Aboul-Fadl, T. Curr. Med. Chem. 2005, 12, 2193.

3. Izquierdo, M. Cancer Gene Therapy 2005, 12, 217.

4. Zaug, A. J.; Been, M. D.; Cech, T. R. Nature 1986, 324, 429.

5. Li, Y.; Breaker, R. R. J. Am. Chem. Soc. 1999, 121, 5364.

6. Breaker, R. R. Nat. Biotechnol. 1997, 15, 427.

7. Santoro, S. W.; Joyce, G. F. Proc. Natl. Acad. Sci. U.S.A. 1997, 94, 4262.

8. Lu, Y. Chem. Eur. J. 2002, 8, 4588.

9. Navani, N. K.; Li, Y. Curr. Opin. Chem. Biol. 2006, 10, 272.

10. Silverman, S. K. Angew. Chem. Int. Ed. 2010, 49, 7180.

11. Breaker, R. R.; Joyce, G. F. Chem. Biol. 1994, 1, 223. 
12. Achenbach, J. C.; Chiuman, W.; Cruz, R. P. G.; Li, Y. Curr. Pharm. Biotechnol. 2004, 5, 312.

13. Baum, D. A.; Silverman, S. K. Cell. Mol. Life Sci. 2008, 65, 2156.

14. Peracchi, A. ChemBioChem 2005, 6, 1316.

15. Santoro, S. W.; Joyce, G. F. Biochemistry 1998, 37, 13330.

16. Fokina, A. A.; Meschaninova, M. I.; Durfort, T.; Venyaminova, A. G.; François, J.-C. Biochemistry 2012, 51, 2181.

17. Yehl, K.; Joshi, J. R.; Greene, B. L.; Dyer, R. B.; Nahta, R.; Salaita, K. ACS Nano 2012, 6, 9150.

18. Rong, W.; Xu, L.; Liu, Y.; Yu, J. P.; Zhou, Y.; Liu, K. L.; He, J. L. Bioorg. Med. Chem. Lett. 2012, 22, 4238.

19. Li, J.; Lu, Y. J. Am. Chem. Soc. 2000, 122, 10466.

20. Kim, H. K.; Rasnik, I.; Liu, J. W.; Ha, T. J.; Lu, Y. Nat. Chem. Biol. 2007, 3, 762.

21. Schlosser, K.; Gu, J.; Sule, L.; Li, Y. F. Nucleic Acids Res. 2008, 36, 1472.

22. Cruz, R. P. G.; Withers, J. B.; Li, Y. Chem. Biol. 2004, 11, 57.

23. Brown, A. K.; Li, J.; Pavot, C. M. B.; Lu, Y. Biochemistry 2003, 42, 7152.

24. Mazumdar, D.; Nagraj, N.; Kim, H.-K.; Meng, X.; Brown, A. K.; Sun, Q.; Li, W.; Lu, Y. J. Am. Chem. Soc. 2009, 131, 5506.

25. Peracchi, A.; Bonaccio, M.; Clerici, M. J. Mol. Biol. 2005, 352, 783.

26. Donini, S.; Clerici, M.; Wengel, J.; Vester, B.; Peracchi, A. J. Biol. Chem. 2007, 282, 35510.

27. Cowan, J. A. Chem. Rev. 1998, 98, 1067. 
28. Hollenstein, M.; Hipolito, C. J.; Lam, C. H.; Perrin, D. M. ACS Combinatorial Science 2013, $15,174$.

29. Cieslak, M.; Szymanski, J.; Adamiak, R. W.; Cierniewski, C. S. J. Biol. Chem. 2003, 278, 47987.

30. Young, D. D.; Lively, M. O.; Deiters, A. J. Am. Chem. Soc. 2010, 132, 6183.

31. Wang, B.; Cao, L.; Chiuman, W.; Li, Y.; Xi, Z. Biochemistry 2010, 49, 7553.

32. Ward, W. L.; Plakos, K.; DeRose, V. J. Chem. Rev. 2014, 114, 4318.

33. Sigel, R. K. O.; Pyle, A. M. Chem. Rev. 2007, 107, 97.

34. Lu, Y. Inorg. Chem. 2006, 45, 9930.

35. Greger, R.; Windhorst, U., Eds. Comprehensive Human Physiology; Springer-Verlag: Berlin-Heidelberg, 1996. 